\title{
Agulhamento seco para alívio da dor em paciente do sexo feminino com hipertrofia bilateral dos músculos masseter e temporal - Relato de caso
}

Dry needling for pain relief in female patient with bilateral hypertrophy of masseter and temporal muscles- Case report

Punción seca para el alivio del dolor em pacientes femininas com hipertrofia bilateral de masetero y músculos temporales- Informe del caso

Déborah Bezerra Sobreira da Silva ORCID: https://orcid.org/0000-0002-7594-4271

Universidade Estadual de Pernambuco, Brasil E-mail: deborahsobreira12@gmail.com

Michele Gomes do Nascimento ORCID: https://orcid.org/0000-0003-2175-7080 Universidade Estadual de Pernambuco, Brasil E-mail: michelepmpe@yahoo.com.br

Hemanuelly Albuquerque dos Anjos ORCID: https://orcid.org/0000-0002-5144-0474 Universidade Estadual de Campinas; Brasil E-mail: hemanuelly.anjos@gmail.com Juliana da Silva Vieira

ORCID: https://orcid.org/0000-0001-6398-3110 Universidade Estadual de Pernambuco, Brasil E-mail: juliana.svieira@upe.br

Douglas José Abreu da Silva Cristovam ORCID: https://orcid.org/0000-0002-9004-3180 Faculdade de Odontologia do Recife, Brasil E-mail: douglasjosecristovam@gmail.com Tomás de Barros Souza ORCID: https://orcid.org/0000-0003-3843-4739 Faculdade de Odontologia do Recife, Brasil E-mail: drtomassouza@gmail.com Caio de Lima Pires

ORCID: https://orcid.org/0000-0003-3178-9537 Universidade Estadual de Pernambuco, Brasil E-mail: caiopires71@ hotmail.com

\begin{abstract}
Resumo
Referido no passado como Síndrome do Minotauro, a hipertrofia do masseter é uma condição relativamente rara, com cerca de 130 casos relatados na literatura, acometendo adultos entre vinte e trinta anos, podendo ser diagnosticado no final da adolescência, sem predisposição por gênero. $O$ paciente pode apresentar entre os achados clínicos uma incapacidade funcional e queixa de desarmonia oclusal, embora na maioria dos casos, a queixa estética é a mais comum. Tem crescimento lento, progressivo, uni ou bilateral e na grande maioria dos casos apresenta ausência de quadro álgico associado. Estudos demostram que a intervenção com o Agulhamento Seco possui benefícios em diversos casos além da dor miofascial, como hemiparesias de membro superior, dedo em gatilho, fibromialgia e disfunções orofaciais. Diante disso o presente estudo teve como objetivo relatar um caso clinico onde a técnica do agulhamento seco foi utilizada para alivio da algia da paciente diagnosticada com hipertrofia dos músculos masseter e temporal, apresentando resultado satisfatório não só no controle da dor como na estética da paciente.
\end{abstract}

Palavras-chave: Hipertrofia; Músculo masseter; Músculo temporal; Agulhamento seco.

\begin{abstract}
Referred to in the past as Minotaur Syndrome, masseter hypertrophy is a relatively rare condition, with about 130 cases reported in the literature, affecting adults between twenty and thirty years, and can be diagnosed in late adolescence, without predisposition by gender. The patient may present among the clinical findings a functional disability and complaint of occlusal disharmony, although in most cases, the aesthetic complaint is the most common. It has slow, progressive, uni or bilateral growth and in the vast majority of cases presents absence of associated pain. Studies show that the intervention with Dry Needling has benefits in several cases besides myofascial pain, such as upper limb
\end{abstract}


hemiparesis, trigger finger, fibromyalgia and orofacial dysfunctions. Therefore, the present study aimed to report a clinical case where the dry needling technique was used to relieve the pain of the patient diagnosed with hypertrophy of the masseter and temporal muscles, presenting satisfactory results not only in pain control but also in the patient's aesthetics.

Keywords: Hypertrophy; Masseter muscle; Temporal muscle; Dry needling.

\section{Resumen}

Conocida en el pasado como Síndrome del Minotauro, la hipertrofia masetero es una condición relativamente rara, con alrededor de 130 casos reportados en la literatura, que afecta a adultos entre veinte y treinta años, y puede diagnosticarse en la adolescencia tardía, sin predisposición por género. El paciente puede presentar entre los hallazgos clínicos una discapacidad funcional y queja de desarmonía oclusal, aunque en la mayoría de los casos, la queja estética es la más común. Tiene un crecimiento lento, progresivo, uni o bilateral y en la gran mayoría de los casos presenta ausencia de dolor asociado. Los estudios demuestran que la intervención con Dry Needling tiene beneficios en varios casos además del dolor miofascial, como la hemiparesia de las extremidades superiores, el dedo en gatillo, la fibromialgia y las disfunciones orofaciales. Por lo tanto, el presente estudio tuvo como objetivo informar un caso clínico donde se utilizó la técnica de punción seca para aliviar la algia del paciente diagnosticado con hipertrofia de los músculos masetero y temporal, presentando resultados satisfactorios no solo en el control del dolor sino también en la estética del paciente.

Palabras clave: Hipertrofia; Músculo masetero; Músculo temporal; Punción seca.

\section{Introdução}

A hipertrofia do músculo masseter é um crescimento excessivo uni ou bilateral do músculo, de etiopatogenia ainda não definida que, na maioria das vezes, gera um desconforto estético e em alguns casos, funcional. Os indivíduos afetados frequentemente exibem um aumento em volume ósseo em região de ramo e ângulo mandibular e o tratamento pode ser através de métodos cirúrgicos ou não cirúrgicos (Rodrigues et al, 2013). Este desenvolvimento muscular exacerbado pode ocorrer uni ou bilateralmente, além de poder estar associado a outros músculos, como por exemplo, o músculo temporal (Tonello et al., 2021; Francisco \& Lopes, 2018).

Apesar de sua etiopatogenia ainda permanecer obscura, alguns autores citam hábitos parafuncionais, como bruxismo e esforço mastigatório excessivo do musculo como algumas das causas possíveis para esta condição, além da perda dentaria. (Rispoli et al, 2008). Pesquisas cientificas também já relacionaram este quadro com pacientes portadores de Disfunção Temporomandibular-DTM (Grazilliano et al, 2016). É importante abordar também, que a hipertrofia do masseter pode se assemelhar a outras patologias, como tumefações e adipoma (Vieira, 2019 e Pinzon et al, 2018)

O primeiro sinal visível desta hipertrofia é a assimetria facial causada pelo alargamento muscular, o que causa um desconforto estético no indivíduo, levando-o a procurar um profissional para avaliar sua situação (Ayhan et al., 2018). O diagnostico inicial é constatado com o auxílio do exame clinico intra e extraoral, onde é realizada a palpação de toda a musculatura facial, além da região das glândulas salivares na busca por possíveis obstruções e suspeita de tumores malignos. Além disto, exames laboratoriais, como a radiografias panorâmica, lateral para ângulo da mandíbula, póstero-anterior de crânio, ortopântomografia, tomografia computadorizada de feixe cônico e a ressonância magnética auxiliarão com análises mais detalhadas da face, para assim observar se existe alguma alteração de desenvolvimento óssea que possa a vir a ser o fator desencadeante para a hipertrofia (Manganello-Souza et al, 2000).

Um importante momento para o diagnóstico é a ativação dos dois músculos através do apertamento dental, onde é possível observar, de fato uma protuberância desta estrutura, deixando-a mais firme ou não provocando necessariamente alterações em sua flacidez (Mandell \& Kayanar, 1994). Segundo Giundice et al. (1992), a hipertrofia dos músculos masseter e temporal pode ser classificada em três tipos, sendo eles:

Tipo I - hipertrofia palpável e visível somente em oclusão forçada.

Tipo II- hipertrofia visível e com ângulo mandibular proeminente.

Tipo III - Hipertrofia visível com exostose no ângulo mandibular 
O tratamento para a hipertrofia dos músculos masseter e temporal pode ser realizado através de métodos mais conservadores, como o auxílio de fisioterapia e a aplicação da toxina botulínica (Vasconcelos et al, 2017; Barbosa \& Barbosa, 2017; Shimaoka et al., 2011; Simão et al, 2014; Santos et al, 2021; Oliveira et al., 2021). Além disso, também podem ser realizados tratamentos mais radicais, como o cirúrgico, que pode consistir na remoção de parte do musculo e, em casos necessários, a também remoção óssea da área afetada (Kessel \& Iserlohn, 1970; Agrawal et al, 2011).

O Agulhamento Seco (DN) é uma técnica que consiste na inserção de uma agulha fina, sólida e filiforme com o objetivo de estimular pontos gatilho, musculatura e tecidos conectivos para modular a dor neuromusculoesquelética e outras alterações como a limitação da amplitude de movimento e incapacidade (Dalpiaz, 2019). Diante disso, o presente caso teve como objetivo relatar um caso de uma paciente com hipertrofia bilateral do masseter e temporal tratada com a técnica do agulhamento seco.

\section{Metodologia}

O estudo tratou de um relato de caso de uma paciente atendida no Hospital da Polícia Militar de Pernambuco. Foi realizado o exame clínico e físico da paciente, além de exames complementares para sustentar o diagnóstico. Após ser diagnosticada com hipertrofia dos músculos masseter e temporal, foi proposto para a paciente o tratamento com agulhamento a seco com a finalidade de controle da dor aguda enquanto o tratamento cirúrgico não fosse realizado.

Todos os aspectos éticos foram respeitados na elaboração deste caso clinico. A paciente foi instruída sobre a necessidade de enriquecer a comunidade acadêmica com novos estudos sobre o caso em questão. A paciente assinou um Termo de Consentimento Livre e Esclarecido para permitir sua participação no estudo. Além disso, os responsáveis pelo desenvolvimento do caso respeitaram a Declaração de Helsinque para cumprimento dos aspectos éticos

Para a sustentação do desenvolvimento do caso de forma correta, foram consultados artigos, livros e periódicos nas bases de dados PUBMED/MEDLINE; LILACS e SCIELO. Os descritores utilizados para a pesquisa foram "hipertrofia"; "musculo masseter"; "musculo temporal" e "agulhamento seco". Foram incluídos relatos de caso que tratavam de hipertrofia do musculo masseter e/ou temporal, independente da data de publicação e tipo de tratamento, além disso também foram incluídos revisões sistemáticas e meta-análises, e estudos que abordavam especificamente a técnica de agulhamento seco, utilizada para guiar o presente caso. Foram excluídos relatos de casos que não se tratavam de hipertrofia dos músculos masseter e temporal.

\section{Relato do Caso}

Paciente, sexo feminino, 45 anos de idade, xantoderma, procurou o serviço odontológico do Hospital da Polícia Militar de Pernambuco -HPMPE, se queixando de dores na região da face, estas sendo mais exacerbadas em momentos de ansiedade e estresse. Também foi relatado pela paciente o hábito de apertamento dentário e episódios de travamento da musculatura, o que vinha the causando dificuldades de abrir e fechar a boca. A paciente também apresentou queixas sobre a estética de sua face, relatando ter tentado disfarçar a hipertrofia muscular através do emagrecimento, mas ao contrário do que a mesma imaginou, a perda de peso apenas evidenciou o crescimento anormal dos músculos.

Através do exame físico foi possível observar hipertrofia nos músculos masseter e temporal, esta característica se apresentando bilateralmente através de um abaulamento significativo da musculatura, como mostra a Figura 1. Foram então solicitados exames complementares para ter uma avaliação mais detalhada do caso e, foram observados nas imagens radiográficas da paciente, a presença de esporão ósseo na região de ramo da mandíbula, bem como uma anormalidade na anatomia do côndilo mandibular. 
Figura 1: Fotografia da paciente na primeira consulta, com hipertrofia bilateral visível dos músculos masseter e temporal. O lado esquerdo mostra alteração mais exacerbada do que o lado direito.

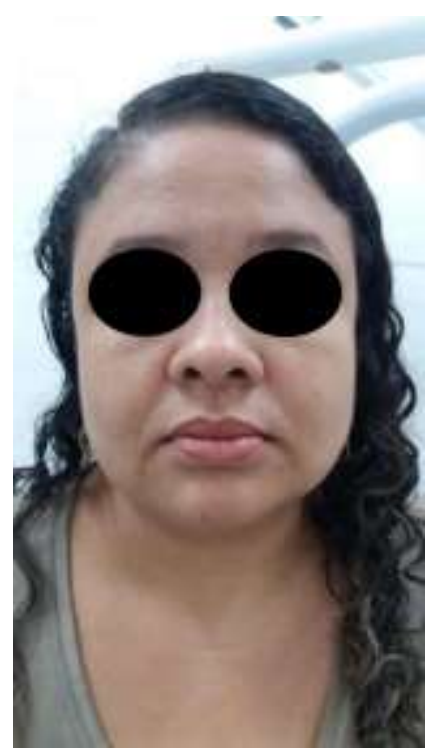

Fonte: Autores.

A paciente foi orientada sobre hábitos e comportamentos que lhe ofertariam mais conforto e evitariam a frequência das crises, além de receber um tratamento de Agulhamento Seco (DN) para controlar o episódio de dor aguda que enfrentava no momento. Ela foi reavaliada em um período de 30 dias após o procedimento de analgesia, relatando melhora na dor e notando discreta melhora no aspecto estético (Figura 2).

Figura 2: Retorno da paciente após 30 dias, relatando melhora significativa da dor e discreta melhora no aspecto estético

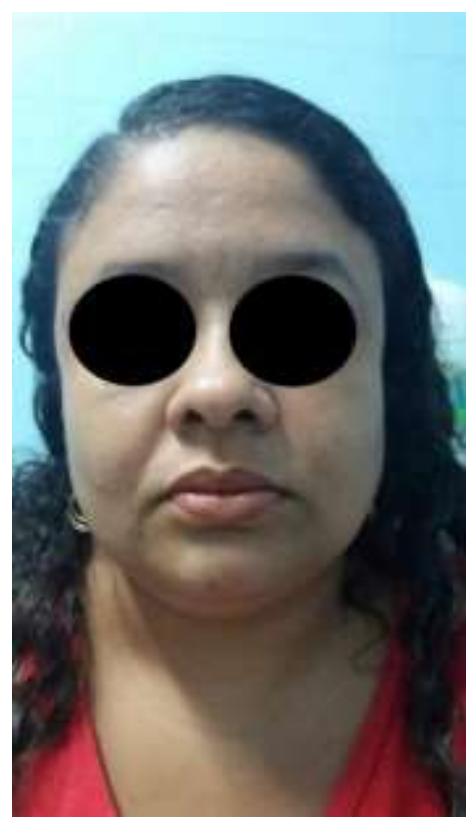

Fonte: Autores.

A paciente relatou desconforto apenas ao passar por situações que causavam ansiedade, mas ainda assim as crises se tornaram menos frequentes. Posteriormente, a paciente foi encaminhada para um Cirurgião-Buco Maxilo Facial indicado pela equipe com o propósito de realizar o tratamento cirúrgico, uma vez que apesar do DN ter demonstrado bons resultados, este 
serve apenas de tratamento complementar, auxiliando na dor da paciente. Dessa forma, para resolução total do caso, a técnica cirúrgica é a mais indicada.

\section{Discussão}

A hipertrofia benigna do musculo masseter é considerada uma patologia rara, que afeta em sua maioria jovens adultos na faixa dos 20-30 anos, não apresentando predileção por sexo (Pinzon et al, 2018; Ferreira, 2019). Geralmente é assintomática, porém esta patologia pode vir a ter a dor entre seus sintomas, sendo esta bem específica e delimitada. Outro sinal que pode estar relacionado com a hipertrofia do masseter é a formação de um esporão ósseo na região do ramo da mandíbula (Caldwell \& Hughes, 1957), no caso em questão, o esporão ósseo foi detectado através de exames radiográficos.

Ainda que o quadro de hipertrofia dos músculos masseter e temporal possam ser revertidos com o auxílio da fisioterapia e de terapêuticas baseadas na aplicação de toxina botulínica, a abordagem cirúrgica ainda é considerada a o método mais assertivo (Oliveira, 2004). O tratamento cirúrgico é tido como padrão ouro, tratando a nível muscular e ósseo, diminuindo as chances de um novo crescimento exacerbado destas estruturas (Grazilliano et al, 2016). No caso relatado, optou-se pelo tratamento cirúrgico devido a bilateralidade e ao envolvimento ósseo presente na paciente, como o esporão ósseo e a alteração anatômica no côndilo mandibular. Entretanto, para controle da dor da paciente enquanto a cirurgia não acontecia, optou-se por realizar a técnica do Agulhamento Seco (DN).

A técnica do agulhamento seco é um procedimento definido como a penetração de uma agulha sólida, realizada sem a introdução de qualquer droga, e tem seu princípio no rompimento mecânico dos pontos-gatilhos miofaciais (PGM) que estão localizados nos músculos esqueléticos, ocasionando a dor. Após a penetração da agulha, ela é estimulada com intuito de se desfazer o nódulo, resultando em um reflexo espinhal involuntário que é a resposta de contração local de fibras musculares afetadas (Griswold et al, 2019).

A intervenção com o DN possui benefícios em diversos casos além da dor miofascial, como hemiparesias de membro superior, dedo em gatilho, fibromialgia e disfunções orofaciais. Quando utilizado para o tratamento da dor miofascial, o DN proporciona alívio dor, aumento do limiar de dor a pressão (LDP) e da amplitude de movimento, melhora no controle motor cervical e melhora de índices de incapacidade, disfunção e qualidade de vida (Dalpiaz, 2019).

Os mecanismos exatos pelos quais o DN possui efeitos ainda não são totalmente esclarecidos e a grande parte do que se sabe é direta ou indiretamente proveniente de estudos sobre acupuntura. Acredita-se que o movimento repetitivo e rápido de inserção da agulha possa estimular fibras aferentes sensitivas de largo diâmetro, levando a inibição do corno dorsal da medula espinhal, bloqueando informações de dor geradas pelos nociceptores ao redor do ponto gatilho (Dalpiaz, 2019).

Apesar da paciente ter referido alivio da dor e leve melhora da estética, o DN é tido como um tratamento auxiliar, sendo a terapia cirurgia mais indicada nesse caso. Realizada da maneira menos invasiva possível, a remoção do músculo hipertrófico e, em alguns casos a ressecção da estrutura óssea afetada podem ser feitas através do acesso intra ou extra oral, ficando a escolha do profissional usufruir da técnica que lhe for mais confortável e lhe proporcionar melhor visibilidade, além de ser favorável a expectativa estética do paciente quanto a futuras cicatrizes. É importante também estudar os riscos trazidos pelo acesso em questão, como é o caso de uma possível lesão no nervo marginal mandibular durante o acesso extra oral (Chee \& Fei, 1989). Devido ao sucesso no controle da dor promovido pelo DN, a paciente seguirá realizando as sessões até o momento cirúrgico.

\section{Conclusão}

A hipertrofia do musculo masseter ainda se trata de uma condição rara, o que faz com que os estudos acerca de sua etiologia, diagnóstico e tratamento ainda sejam pouco encontrados. O Agulhamento Seco (DN) é uma boa solução para controle da dor promovida pela hipertrofia, entretanto, o tratamento cirúrgico ainda se configura como padrão ouro para resolução dessa 
patologia, sendo assim o DN se configura apenas como tratamento auxiliar no alivio dos sintomas, não promovendo a resolução total do caso. É de suma importância que aja interesse da classe odontológica por buscar maior conhecimento sobre esta patologia, para além de ofertar um melhor prognostico aos pacientes acometidos por esta hipertrofia muscular, saber também diferenciar outras doenças e expandir o conhecimento odontológico. Além disso, se faz necessário o desenvolvimento de novos estudos sobre o caso, que devem levar em consideração o agulhamento seco como uma alternativa para promover um melhor conforto para o paciente.

\section{Referências}

Agrawal, G., Singh, S., Shivamurthy, D., \& Varghese, D. (2011). Tratamento cirúrgico da hipertrofia massetérica e retrognaismo mandibular. Nat Journ of Maxillofa Surg, 2(1), 96-99

Ayhan M.; Isler S. C., \& Kasaposh C (2018). Combinação de tratamentos médicos e cirúrgicos para hipertrofia de masseter. Caso Represent Dent

Barbosa, C. M. R., \& Barbosa, J. R. A. (2017). Toxina Botulínica em Odontologia. Elsevier.

Caldwell J.B.\& Hughes K.W (1957). Hypertrofhy of the masseter muscles and mandible: report a case. J Oral Surg, 15(4), 329-331

Chee F.L \& Fei Y.J. (1989). Surgical correction of masseter muscle hypertrophy by an intraoral approach. J. Oral Maxillofac. Surg. 47:883-5.

Dalpiaz A. (2019). Agulhamento á seco e fotomodulação no tratamento da dor miofascial em trapezio superior de mulheres. Universidade Federal de Santa Catarina, Araraguá

Ferreira, G. M. (2019). Toxina botulínica: efeitos indesejados nos músculos mastigatórios. Rev face magazine. https://facemagazine.com.br/efeitos-indesejados da-toxina-botulinica-nos-musculos-mastigatorios

Giundice M.; Marra A.; Barba A.; Passariello N.\& D’Onofrio F (1992). Ipertrofia dei masseteri: un raro caso, associato a cardiopatia ipertrofica. Minerva Stomatl. 41:535-42.

Graziliano P., et al. (2016) Hipertrofia bilateral dos músculos masseter e temporal: Nossos quinze pacientes e uma revisão de literatura. Eur Rev Med Pharmacol Sci, 1(20), 7-11

Griswold D., et al. (2019). The effectiveness of superficial versus deep dry needling or acupuncture for reducing pain and disability in individuals with spinerelated painful conditions: a systematic review with meta-analysis. Journal of Manual \& Manipulative Therapy, 1-13.

Kessel, L. \& Iserlohn, R. A. D. C. (1970). Benign bilateral masseteric hypertrophy with temporal muscle involvement. Oral Surg. Oral Med. Oral Pathol. 30:450 Mandell L.\& Kayanar A. (1994). Masseteric Hipertrofy. NYSDJ, 7(60), 44-47

Manganelo-Souza D., et al. (2000). Hipertrofia do músculo masseter. Rev Soc Bras de Cir Plast, 15(1), 45-54.

Oliveira D. M., et al. (2004) Hipertrofia do masseter: relato de caso. Rev Cir Trauma BucoMaxiloFacial. 4(1), 31-38

Oliveira, M. E. S. G. de; Friggi, S. L. M. \& Shitsuka, C. (2021) Occlusal splint associated with auxiliary therapies to control the painful symptomatology of the bruxist patient. Research, Society and Development, 10(14), e131101421751. https://rsdjournal.org/index.php/rsd/article/view/21751.

Pinzon, N. A., Rodriguez, E. A. S., Goulart, D. R., Orlate, S., Farah, G. J \& Filho, L. I. (2018). Toxina botulínica para o tratamento de distúrbios temporomandibulares. Int. J. Odontostomat. 12(2), 103-109

Rodrigues D.C., et al. (2013). Hipertrofia Unilateral do Músculo Masseter: Relato de Caso. Rev. cir. traumatol. buco-maxilo fac. 13(4)

Rispoli, D. Z., Camargo, P. M., Pires, Jr. J. L., Fonseca, V. R., Mandelli, K. K., \& Pereira, M. A. C. (2008). Hipertrofia benigna do músculo masseter. Rev bras otorrinolaringol. 74(5), 790-93

Santos, M. E. S.; Santos, T. A. dos; Nascimento, F.; Martins, V. da M \& Dietrich, L. (2021) Botulinic toxin for masseter hypertrophy. Research, Society and Development, 10(13), e580101321412, 10.33448/rsd-v10i13.21412. https://rsdjournal.org/index.php/rsd/article/view/21412

Shimaoka, A. M., Andrade, A. P., \& Carvalho, R. C. R. (2011). O uso da toxina botulínica na odontologia. (5), 1-3.

Simão, N. R., Borba A. M., Silva, A. L. F., \& Almeida N. S. (2014). Hipertrofia benigna do músculo masseter -relato de caso. Rev. Assoc. Paul Cir. 68 (4), 351 5.

Tonello C.; Bastos E.O.; Feitosa L.B. \& Alonso N. (2021). Hipertrofia idiopática bilateral de músculos temporal e masseter. Brazilian Applied Science Review, Curitiba, (1), 372-377

Vasconcelos, A. F. M., Filho, G. C. S., Cavalcante, A. B, \& Vasconcelos, B. C. E. (2017). Toxina botulínica nos músculos masseter e temporal: considerações farmacológicas, anatômicas e clínicas. Rev. Cir. Traumatol Buco-Maxilo-Facial. 16(2), 50-54 